\title{
Status and prospects for hadron production experiments
}

\section{M.G.Catanesi*}

Istituto Nazionale di Fisica Nucleare, Bari, Italy

E-mail: gabriella.catanesi@cern.ch

\begin{abstract}
Hadron production measurements for neutrino experiments is a well established field at CERN since the '70s. Precise prediction of atmospheric neutrino fluxes, characterization of accelerator neutrino beams, quantification of pion production and capture for neutrino factory designs, all of these would profit from hadron production measurements. In recent years, interest in such studies was revived and new generation of low-energy (from 3 to $400 \mathrm{GeV}$ ) hadron production experiments were built or proposed. Such experiments all share a basic design, consisting in the presence of open-geometry spectrometers, as close as possible to full angular coverage, and aiming at full particle identification. Many new results are now provided by Harp in the very low energy range ( 3 to $15 \mathrm{GeV} / \mathrm{c}$ ). NA61 at CERN and MIPP at Fermilab are taking data and in the next years will explore the medium energy range (up to $400 \mathrm{GeV} / \mathrm{c}$ ). Finally at LHC energies for the first time thanks to the TOTEM experiment, it will be possible to measure with unprecedented precision the total cross section beyond $1 \mathrm{TeV} / \mathrm{c}$.
\end{abstract}

10th International Workshop on Neutrino Factories, Super beams and Beta beams June 30 - July 52008

Valencia, Spain

\footnotetext{
${ }^{*}$ Speaker.
} 


\section{Introduction}

Hadron production data are relevant in several branches of neutrino physics.

The study of atmospheric neutrinos provides strong evidence for neutrino oscillations[1]. At the same time, to quantitatively understand this phenomenon, several accelerator-based neutrino experiments are being built, and new types of neutrino beams are being designed. In either cases, detailed knowledge of the hadron cross sections at the relevant energies is now considered a must. The design of alternative neutrino beams would profit a lot from a more detailed knowledge of the hadron production cross-sections. The optimization of the figure of merit (and the cost) of the so-called neutrino factory [2] (neutrino beam produced by decays in a muon storage ring) depends on the production and collection efficiency of pions at the target station, and therefore requires a systematic study of pion production cross sections at several energies (from a few $\mathrm{GeV}$ to $24 \mathrm{GeV}$, depending on design choices) and with several target materials.

The calculation of atmospheric neutrino fluxes is dominated by the knowledge of the primary cosmic ray flux, of the hadron production in the interaction of the primaries with target air nuclei, and of the subsequent decay chains. Most of the uncertainty comes from the limited understanding of hadron interactions. Different Monte-Carlo simulations, depending on the model they are based on ([3, 4, 5] as examples), provide estimates which can differ by as much as $25 \%$. The relevant energy range for primary particles is, in this case, from a few $\mathrm{GeV}$ to a few $100 \mathrm{GeV}$, while target material should be as close as possible to the constituents of the atmosphere, namely $\mathrm{N}_{2}$ and $\mathrm{O}_{2}$.

\section{Historical perspective}

Since the 70's, in order to make a reliable prediction of the neutrino flux, the community has always been committed to measure in ancillary experiments the hadron production cross-sections, the reason being that neutrino beam composition relies on these measurements.

This first generation of hadron production experiments was mostly based on measurements of particle yield along instrumented beam lines, manly using existing facilities. They were all single arm spectrometers with a small phase space coverage in the forward region $(<150 \mathrm{mrad})$, and characterized by low statistic and/or limited number of data points.

The overall scale error, arising from the uncertainties in the spectrometer acceptances and in the absolute calibration of the primary proton beam intensity, was estimated to be $15 \%$.

Several measurements of this kind were done in the past. We should mention in the low energy range (around $20 \mathrm{GeV} / \mathrm{c}$ ) of primary proton momentum, the experiments quoted as "Allaby" [6] and "Eichten" [7]. At higher energy we should quote the NA20 spectrometer [8],that performed a secondary particle energy scan in the range 60-300 GeV/c, and the more recent (1996) SPY experiment [9].

\section{HARP}

HARP at the CERN PS [10][11] was the first hadron production experiment designed on purpose, combining a full phase space acceptance with low systematic errors and high statistic. 
HARP performed extensive measurements of hadron production cross sections and secondary particle yields in the energy range $3-15 \mathrm{GeV}$ over the full solid angle, using a large set of cryogenic and solid (thin and thick) targets. A forward spectrometer covers polar angles up to $250 \mathrm{mrad}$ which is well matched to the angular range of interest to calculate the properties of conventional neutrino beams.In particular, it matches the acceptance of the K2K and MiniBooNE beam lines. The particle identification is performed with a combination of time-of-flight, Cherenkov, and calorimeter information. In the large-angle region a TPC positioned in a solenoidal magnet is used for tracking and the particle identification is performed with the dedx in the TPC and the time-of-flight measurements with RPCs. The large-angle spectrometer acceptance is optimized for the measurement of pions relevant to the production of muons beams in future neutrino factories; it covers $90 \%$ of the pions accepted in the focusing system of a typical design. By making use of a fast readout (event rate $2.5 \mathrm{KHz}$ ), very demanding (and unprecedented at that time) for a TPC, the HARP detector was able to collect few millions of events per setting (a setting is a combination of target type and material, beam energy and polarity) insuring small statistical errors.

\section{Results obtained with the HARP forward spectrometer}

\section{1 $\pi$ production using the $\mathrm{K} 2 \mathrm{~K}$ and MiniBoone}

The $\mathrm{K} 2 \mathrm{~K}$ experiment is most sensitive to uncertainties in the predicted $v$ spectrum in the energy range between 0.5 and $1 \mathrm{GeV}$. The distortion of the spectrum measured with the far detector is predicted to be maximal in this range according to the $v$ oscillation parameters measured in atmospheric $v$ experiments. The transmission properties of the beam line in the K2K experiment are such that they match very well the HARP forward spectrometer acceptance. The measurement of $\pi$ production for the $\mathrm{K} 2 \mathrm{~K}$ experiment using a $5 \% \lambda \mathrm{Al}$ target and incident protons of $12.9 \mathrm{GeV} / \mathrm{c}$ momentum was published [12]. In Fig.1(left) a ratio is shown between the $v$ flux measured at the near and at the far detector as a function of the $v$ energy. A comparison of the errors on the near/far ratio before and after the inclusion of the HARP results shows a reduction by a factor of two. The neutrino flux calculation for final disappearance analysis of $\mathrm{K} 2 \mathrm{~K}[13]$ is based on the HARP pion production data.

The case for the MiniBooNE measurement is equally compelling. Here too the $v$ flux comes predominantly from the $\pi$ decay into $\mu+v$. Again in this case the HARP experiment covers the relevant range, $0.75-6.5 \mathrm{GeV} / \mathrm{c}$ in energy and 30-210 mrad in angle (more than $80 \%$ of the phase space). The cross section results published in [14] have a direct impact on the prediction of $v$ fluxes at Miniboone. The Miniboone Results [15] are based on a parametrization of the HARP $\pi^{+}$and $\pi^{-}$cross sections.

\subsection{Atmospheric neutrino fluxes and extended air showers}

The measurements of the cross-section of $\mathrm{p}-\mathrm{C}$ interactions have direct relevance for the precise prediction of atmospheric neutrino fluxes and extended air showers (EAS) modeling. Carbon is an isoscalar nucleus and so are nitrogen and oxygen, so the extrapolation to air is the most straightforward. The existing data for carbon target at low energies are very limited. The measurement of the double-differential production cross-section of $\pi^{ \pm}$in the collision of $12 \mathrm{GeV} / \mathrm{c}$ protons with 

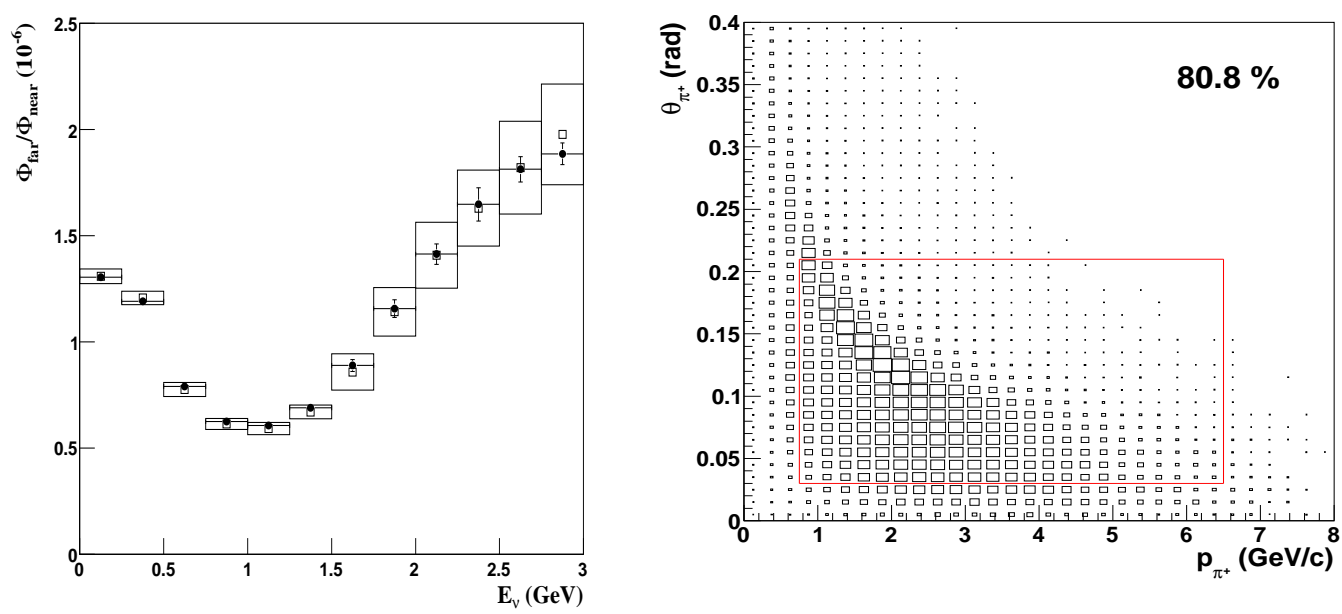

Figure 1: Near to far ratio in the $\mathrm{K} 2 \mathrm{~K}$ experiments measured by the $\mathrm{K} 2 \mathrm{~K}$ MonteCarlo (boxes) and results from the Harp experiment (errored points)(left); MiniBoone Phase space: inside the box the region covered by HARP(right).

a thin 5\% $\lambda_{\mathrm{I}}$ carbon target has been performed using the HARP forward spectrometer. A systematic error analysis has also been done yielding integral errors (statistical + systematic) of $6.1 \%$ and $7.0 \%$ for $\pi+$ and $\pi$ - in p-C interactions, $10.2 \%$ and $8.5 \%$ for $\pi+$ and $\pi$ - in $\pi+-\mathrm{C}$ interactions, $6.5 \%$ and $8.2 \%$ for $\pi+$ and $\pi$ - in $\pi$-C interactions, and an overall normalization error of $2 \%$ for the proton beam and 3\% for the pion beams [16]. More recently for the first time also results using $\mathrm{N}_{2}$ and $\mathrm{O}_{2}$ cryogenic targets have been published [17]. In particular those results allows to confirm that the common hypothesis that $\mathrm{p}-\mathrm{C}$ data can be used to predict the $\mathrm{N}_{2}$ and $\mathrm{O}_{2}$ pion production cross-sections.

\section{Results obtained with the HARP Large Angle spectrometer}

One of the main motivations of the HARP experiment is the measurement of the yields of positive and negative $\pi \mathrm{s}$ for a quantitative design of a proton driver and a target station in a future $v$-factory. The variables affecting the $\pi$ production are incident proton beam energy, target material and target geometry (diameter and length). In order to achieve the highest number of potentially collected $\pi \mathrm{s}$ of both charge signs per unit of energy a $\pi$ production measurement should give the information necessary to optimize both proton beam energy and target material.

At the moment, a CERN scenario for a $v$-factory foresees a $3 \mathrm{GeV} / \mathrm{c}$ or $5 \mathrm{GeV} / \mathrm{c}$ proton linac with a high- $Z$ target material [18]. Even if this is not the only one studied scenario, in most of the cases high- $Z$ materials are proposed as targets. For this reason it was decided to first analyze a series of settings taken with a range of different beam momenta (from 3 to $12 \mathrm{GeV} / \mathrm{c}$ ) incident on a Tantalum ( $\left(5 \% \lambda_{\text {int }}\right)$ target [19]. For this purpose the produced $\pi \mathrm{s}$ are best measured in the largeangle spectrometer, covering the full momentum range of interest for production angles above 0.35 $\mathrm{rad}$. As an indication of the overall pion yield as a function of incoming beam momentum, the $\pi+$ and $\pi$ - production cross-sections in $\mathrm{p}$-Ta interactions were integrated over the full HARP kinematic 

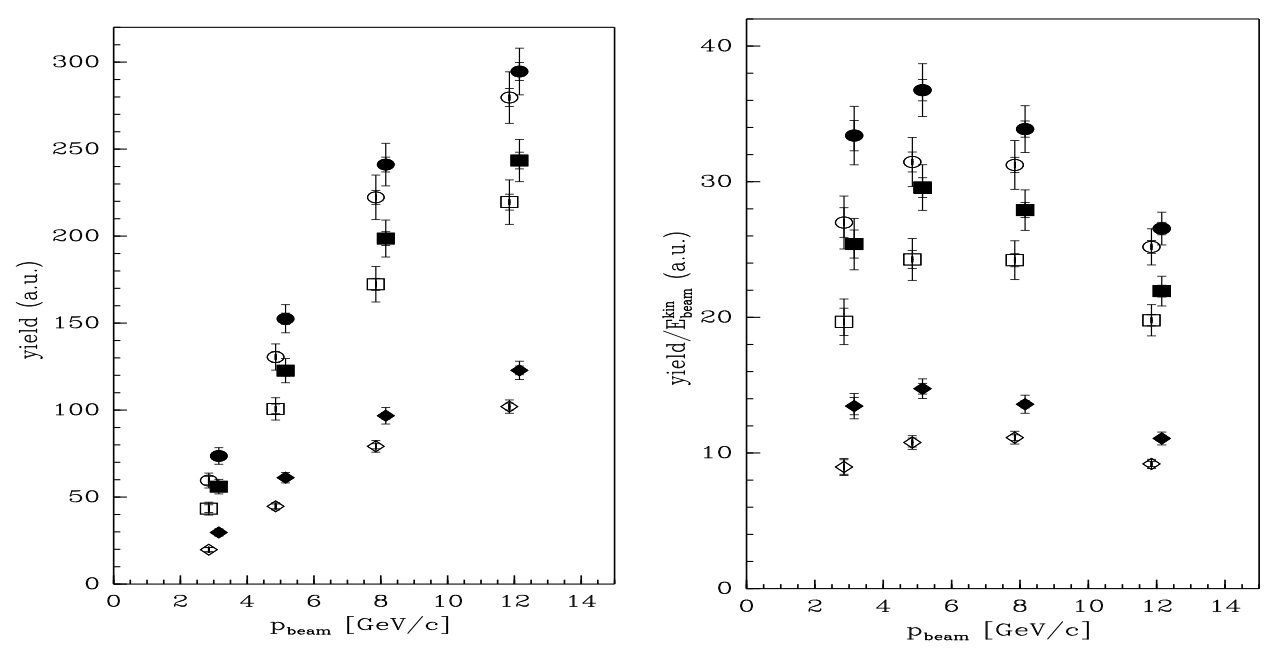

Figure 2: Prediction of the $\pi+$ (closed symbols) and $\pi$ - (open symbols) yield from a tantalum target as a function of incident proton beam momentum for different designs of the neutrino factory focusing stage. Shown are the integrated yields (left) and the integrated yields normalized to the kinetic energy (right). The circles indicate the integral over the full HARP acceptance, the squares are integrated over $0.35 \mathrm{rad}<\theta<$ $0.95 \mathrm{rad}$, while the diamonds are calculated for the restricted angular range and $250 \mathrm{MeV} / \mathrm{c}<\mathrm{p}<500 \mathrm{MeV} / \mathrm{c}$.

range. The results are shown in Fig. 2. The integrated yields are shown in the left panel and the integrated yields normalized to the kinetic energy of the incoming beam particles are shown in the right panel. The outer error bars indicate the total statistical and systematic errors. It is shown that the pion yield increases nearly linearly with momentum and that in our kinematic coverage the optimum yield is between $5 \mathrm{GeV} / \mathrm{c}$ and $8 \mathrm{GeV} / \mathrm{c}$. To show the trend, rates within restricted ranges are also given: the latter may be most representative for the neutrino factory. Of course this analysis only gives a simplified picture of the results. These calculations should be completed with more realistic kinematical cuts in the integration.

Similar analyses have been performed for the $\mathrm{Be}, \mathrm{C}, \mathrm{Al}, \mathrm{Cu}, \mathrm{Sn}$ and $\mathrm{Pb}$ targets using the same detector [20, 21, 22]. They allow, for example, to study the integrated pion yield as a function of beam momentum, its A-dependence, the $\pi-/ \pi+$ ratio etc. with a reduced systematic uncertainty. The fact that these data are taken with the same apparatus and analysed using the same methods improves the precision of the relative comparisons. The beam energy dependence of the yields is clearly different in the low $A$ data compared to the high $A$ data. For example, the dependence in the $\mathrm{p}-\mathrm{C}$ data is much flatter than the tantalum data with a saturation of the yield between 8 $\mathrm{GeV} / \mathrm{c}$ and $12 \mathrm{GeV} / \mathrm{c}$. Also the $\pi+$ and $\pi$-production yields exhibit a different behavior. In the $\mathrm{p}-\mathrm{C}$ interactions the $\pi+/ \pi$ - ratio increases with increasing beam momentum, and a change of slope of the ratio as function of secondary momentum is visible. These features are different from the ones observed in the p-Ta data [19], where the ratio is closer to unity and a smaller variation with beam momentum is observed. Finally in [22] an exhaustive comparisons between the Hadronic production models describing this energy range and the corresponding data can be found. 

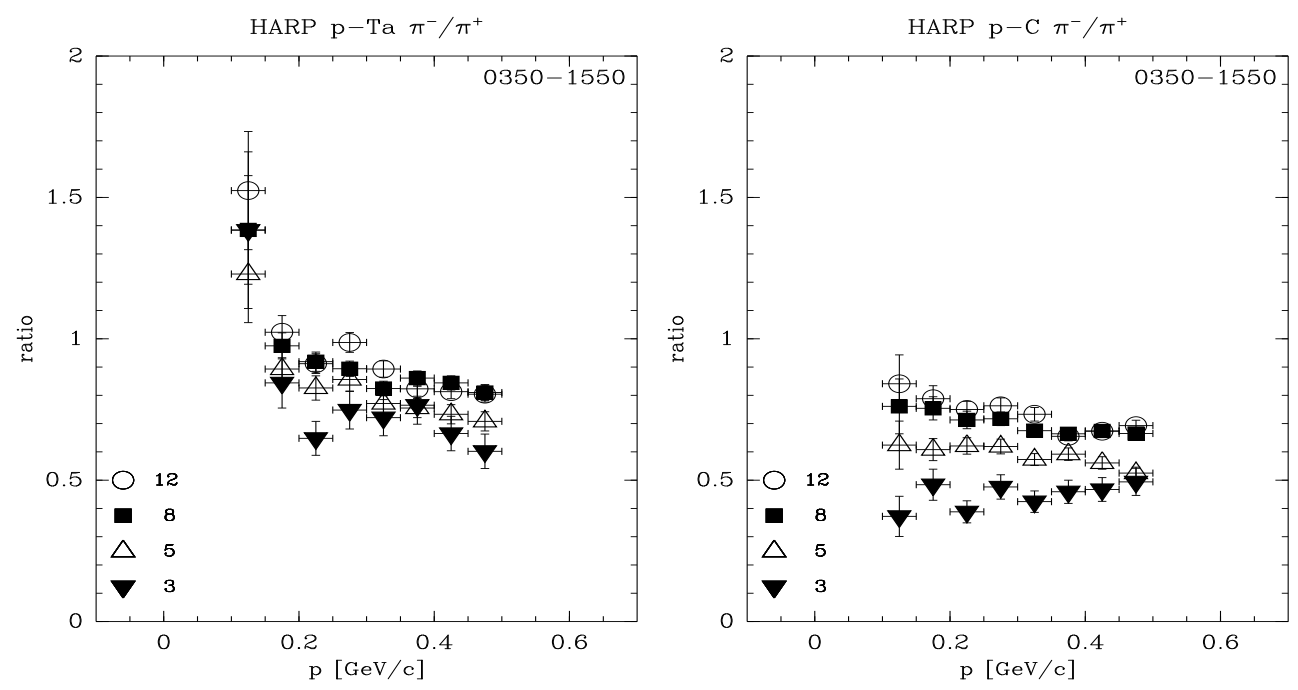

Figure 3: The ratio of the differential cross-sections for $\pi$ - and $\pi+$ production in $\mathrm{p}-\mathrm{Ta}$ (left panel) and $\mathrm{p}-\mathrm{C}$ (right panel) interactions as a function of secondary momentum integrated over the forward angular region (shown in mrad). The results are given for all incident beam momenta (filled triangles: $3 \mathrm{GeV} / \mathrm{c}$; open triangles: $5 \mathrm{GeV} / \mathrm{c}$; filled rectangles: $8 \mathrm{GeV} / \mathrm{c}$; open circles: $12 \mathrm{GeV} / \mathrm{c}$ ).

\section{NA49/NA61 and MIPP}

\subsection{NA49/NA61}

The NA49 experiment is a large acceptance hadron spectrometer at the CERN-SPS for the study of the hadronic final states produced by collisions of various beam particles (including nuclei) of different energies on a variety of fixed targets.

The main tracking devices are four large volume Time Projection Chamber (TPCs). The NA49 TPCs allow precise measurements of particle momenta and type, and are supplemented by two time of flight (TOFs) detector arrays and a set of calorimeters for additional particle-ID.

An upgrade of the NA49 detector was approved at CERN in June 2007[23]. It mainly consists in the replacement of the old TPC read-out electronic in order to seed-up the data acquisition rate of a factor 10, in order to match the demand for high-precision measurements. The proposed physics program include measurements of hadron production in hadron-nucleus interaction needed for neutrino (T2K[24]) and cosmic-ray experiments (Auger[25], KASCADE [26])

The T2K experiment will study oscillation of an off axis neutrino beam between the J-Parc accelerator and the Super-Kamiokande detector. Its neutrino beam is produced from a primary $50 \mathrm{GeV} / \mathrm{c}$ proton beam $(30 \mathrm{GeV} / \mathrm{c}$ in a first phase) hitting a Carbon target.

The T2K results will not be statistics limited, so $\mathrm{n}$ order to achieve the required precision be control the systematics will be a must [27]. Following the HARP example, NA61 will measure the primary proton-Carbon cross section at $50 \mathrm{GeV} / \mathrm{C}(30 \mathrm{GeV} / \mathrm{c})$ using a replica of the $\mathrm{T} 2 \mathrm{~K}$ target and several Carbon targets of various thickness. A first pilot run (protons $30 \mathrm{GeV}$ on Carbon target) was successfully performed during October 2007 and the corresponding data analysis is ongoing.

It is well known in cosmic ray physics that at high energy the measurements are based on the analysis of secondary particle showers produced in the atmosphere. The interpretation in terms 
of primary particle type and energy relies strongly on hadron production models. Only recently it has been realized that interactions in the energy range up to few hundred $\mathrm{GeV}$ give rise to large uncertainties. To cover the lack of measurements in this energy range NA61 plans to collect the following sample of data:

- $p-C 30,100,250,400 \mathrm{GeV}$

- $\pi_{+}-C 30,100,250 \mathrm{GeV}$

- $\pi_{-}-C 30,100,250 \mathrm{GeV}$

The Carbon target was chosen due to the similarities of the secondary particle distributions in p-C and p-Air interactions.

\subsection{MIPP}

The E907 Main Injector Particle Production (MIPP) experiment at Fermilab is a full acceptance spectrometer with excellent particle identification capabilities. MIPP has collected $15 \times 10^{6}$ events of p's, $\pi$ and K's at various momenta (from 5 to $120 \mathrm{GeV}$ ) on several targets including beryllium and carbon. In particular, MIPP has collected hadron production data on a spare NuMI target using $120 \mathrm{GeV} / \mathrm{c}$ protons from the Main Injector. Recently preliminary results of particle production ratios of $\pi_{+} / \pi_{-}, \mathrm{K}+/ \pi_{+}, \mathrm{K}-/ \pi_{-}$, and $\mathrm{K}-/ \mathrm{K}+$ in bins of longitudinal and transverse momentum for thin and thick targets start to be available. A comparison has also been made to the particle production ratios as determined from the MINOS re-weighted neutrino energy spectra in the near detector showing a good agreement up to $50 \mathrm{GeV} / \mathrm{c}[28]$.

\section{The high energy frontier:TOTEM}

Totem[29] is the only LHC experiment that will explore the forward region at pseudorapidity larger than 3.1 The main goal is the measurement of the total and elastic cross-section at $14 \mathrm{TeV}$ and the study of diffractive physics in the forward region. The experiment is approved and funded and will start the data taking end 2007.

The total cross section beyond $1 \mathrm{TeV} / \mathrm{c}$ will be measured with the unprecedented precision of $1 \%$ using the luminosity independent method based on the simultaneous detection of elastic scattering at low momentum transfer and of the inelastic interactions. Protons scattered at very small angles in elastic or quasi-elastic reactions will be measured in telescopes of silicon detectors enclosed in Roman Pots, placed on both sides of the intersection regions. Inelastically produced secondaries will be measured by a forward inelastic detector covering the region $3<\eta<7$ with full azimutal acceptance. This last detector will measure the overall rate of inelastic reactions.

Totem shares the interaction point with the CMS experiment, and a common Physics TDR [30] by the two collaborations showing an extensive common physics program was discussed and recently approved by the LHCC Committee at CERN. This program will include for the first time the measurement of the very forward energy flux essential for a better understanding of the cosmic ray events. 


\section{References}

[1] Y. Fukuda (SuperKamiokande Collaboration), B433 (1998) 9; 81 (1998) 1562.

[2] A.Blondel et al. ECFA/CERN Studies of a European Neutrino Factory Complex CERN-2004-002 ECFA-04-230.

[3] T.K.Gaisser et al.,Phys.Rev.D54 (1996)5578.

[4] M.Honda,Nucl.Phys.B77 (1999)140.

[5] R. Engel Nucl.Phys.B (Proc.Suppl.) 122, 40 (2003)

[6] J.V.Allaby et al.,CERN report 70-12 (1970).

[7] T.Eichten et al.,Nucl.Phys.B44,(1972)333.

[8] H.W. Atherton et al.,CERN report 80-07 (1980).

[9] G. Ambrosini et al.,Eur. Phys. J. C10 (1999) 605-627.

[10] M.G.Catanesi et al., CERN-SPSC-99-35 (1999).

[11] M.G. Catanesi et al.,NIM A571 (2007) 527.

[12] M.G.Catanesi et al Nucl.Phys.B732:1-45,2006.

[13] E. Aliu et al. Phys. Rev. Lett. 94 (2005) 081802

[14] M.G. Catanesi et al. E.P.J. C52(2007) 29.

[15] A.A. Aguilar et al. Phys.Rev. Lett. 98, 231801 (2007)

[16] M.G. Catanesi et al. Astropart.Phys.29:257-281,2008.

[17] M.G. Catanesi et al. Astropart.Phys.30:124-150,2008.

[18] M. Apollonio et al., Oscillation Physics with a Neutrino Factory CERN TH2002-208.

[19] M.G. Catanesi et al. Eur.Phys.J.C51:787-824,2007.

[20] M.G. Catanesi et al. Eur.Phys.J.C53:177-204,2008.

[21] M.G. Catanesi et al. Eur.Phys.J.C54:37-60,2008.

[22] M.G. Catanesi et al. Phys.Rev.C77:055207,2008.

[23] N. Antoniou et al..CERN-SPSC-P-330, Nov 2006. 90pp.

[24] Y. Itow et al., "The JHF-Kamioka neutrino project” hep-ex/0106019.

[25] T. Antoni et al. NIM A 513, 490 (2003).

[26] J. Abraham et al. NIM A 523, 50 (2004).

[27] M.H. Ahn et al. Phys.Rev.D74:072003,2006.

[28] D.G.Michael et al. Phys.Rev.Lett.97,191801,2006.

[29] G. Anelli et al. JINST 3:S08007,2008.

[30] M. Albrow et al. "Prospects for Diffractive and Forward Physics at the LHC": CERN-LHCC-2006-039. 